\title{
Coding Verbal Interactions In A Prototype Future Force Command And Control Simulation
}

\author{
Paula J. Durlach \\ U.S. Army Research Institute \\ Laticia D. Bowens and John L. Neumann \\ University of Central Florida \\ Consortium Research Fellows Program \\ Thomas J. Carnahan \\ Western Kentucky University \\ Consortium Research Fellows Program
}

\section{Simulator Systems Research Unit \\ Stephen L. Goldberg, Chief}

\begin{abstract}
U.S. Army Research Institute for the Behavioral and Social Sciences 5001 Eisenhower Avenue, Alexandria, Virginia 22304-4841
\end{abstract}

\section{January 2004}

Army Project Number 20262785A790
Personnel Performance and Training Technology

Approved for public release; distribution unlimited 